\title{
WAR WOUNDS OF THE INTESTINES AND THEIR TREATMENT.
}

By CECIL P. G. WAKELEY, D.Sc., F.R.C.S., F.R.S.E., F.A.C.S., F.R.A.C.S, (Senior Surgeon and Director of Surgical Studies, King's College Hospital; Consulting Surgeon to the Maudsley Hospital and to the Royal Navy; Temporary Surgeon Rear-Admiral.)

Although most surgeons are familiar with cases of rupture of the abdominal viscera due to contusions of the abdominal wall resulting from kicks from horses, falls from scaffolding or motor car accidents, yet there are some who have no experience of abdominal war wounds. The commonest structure to be injured in war wounds of the abdomen is the small intestine and if other structures are not damaged and the case is operated upon within six hours there is a fifty per cent. recovery rate. The time factor is a most important one in abdominal injuries and every effort should be made to get the man to a casualty clearing station with all speed. In the last war it was proved conclusively that the limit in successful cases was forty-eight hours for a resection of the small intestine and thirty-six hours for a suture of the colon.

\section{SYMPTOMATOLOGY.}

The symptomatology of war wounds of the abdomen varies considerably. As a rule pain is a fairly constant symptom, but there are many recorded cases in which men have had their abdomens ripped open by bullets and have not experienced pain or exhibited much shock. The pain is often intense and commences from the moment of infliction, and it can be likened to the pain of a perforated gastric ulcer. In wounds of the lower part of the abdomen if the lumbar plexus is involved there may be referred pain in the leg and this may be all that the wounded man complains about, no notice being taken of the actual entrance wound itself.

The appearance of the patient may be fallacious but as a rule a man hit in the abdomen looks ill, and blanched; however some cases present almost a normal appearance but the pulse rate will nearly always give the key to the actual situation. When there is a rapid pulse or a rising pulse operation is always indicated. Patients with a pulse rate of I2O or over have little chance of recovering, yet it is interesting and gratifying to find that Gordon Taylor reported a series of 34 abdominal wounds with pulses over I24 with I4 recoveries, and a second series of 32 cases with pulse rates of over I4O in which I2 recovered. Such records as these should be a great encouragement to surgeons in the forces of to-day. The rapid pulse is invariably due to hæmorrhage which may be said to be the greatest enemy of the surgeon. The hæmorrhage may come from various sources; the abdominal wall, the wall of the bowel, the mesenteric vessels, the omentum, or vessels in the retroperitoneal tissues. Hæmorrhage occurring in the retroperitoneal tissues is probably the worst type the surgeon may encounter, because there is rarely any indication as to the source of the bleeding. It may come from the renal vessels or even the inferior vena cava. The retroperitoneal hæmatoma may be so great that it raises the peritoneum of the posterior abdominal wall to such an extent that it almost approximates the anterior abdominal wall. On opening the abdomen in such cases the surgeon appears to be dealing with an enormous purple intra-abdominal tumour, and once seen such a condition is never forgotten. As the surgeon can never be certain as to the origin of the hæmorrhage in these retroperitoneal hæmatomata it is wiser to leave well alone and establish drainage should sepsis supervene subsequently.

Rigidity although a fairly constant symptom may occasionally be absent. It is well known that injuries of the thorax, especially if low down, may cause superficial abdominal tenderness, and it is always a wise precaution to examine the thorax 
thoroughly as well as the abdomen. As a rule if the wound is limited to the abdominal wall the tenderness is quite local. Extensive rigidity may be due to the fact that other abdominal viscera are injured or that there is a considerable amount of blood in the abdomen. Rigidity may be more marked in some cases than in others and may vary at hourly intervals, and morphia in moderate doses seems to have little effect upon it.

Tenderness as one would expect varies according to the amount of intraabdominal damage, but as a working rule it may be said that the maximum tenderness coincides with the position of the perforation or perforations of the gut.

Although in some cases the history of the injury cannot be obtained owing to the shocked condition of the patient yet it is always valuable, and important information may be forthcoming. Also the clinical examination should not be neglected, but here again the condition of the patient may be so bad that anti-shock measures must be adopted before any extensive clinical examination can be carried out. Surgical common sense and sound judgment may save many a life.

\section{RADIOGRAPHY.}

The value of Radiography in war injuries of the abdomen is limited and whether it is made use of or not must depend on the condition of the patient. To $\mathrm{X}$-ray a severely shocked patient where the time factor is all important only demonstrates a lack of surgical commonsense. However, in other cases where the condition of the patient warrants it, an X-ray examination may provide useful information regarding the size and situation of the missile.

\section{TREATMENT.}

As the majority of cases with abdominal injuries exhibit a definite shock syndrome it is important that all measures should be taken to combat this. As every perforating wound of the abdomen probably means a perforating wound of the intestines or other viscus, operation is indicated in every case.

\section{Treatment of Shock.}

Shock must be treated before operation and warmth, morphia and blood transfusion will combat this more than any other measure. Blood transfusion should not be performed until the patient is actually on the operating table, because it is important to stop any internal hæmorrhage before more blood is put in circulation, or the majority of the transfusion may only contribute to making a retroperitoneal hæmatoma more extensive. However, every case must be judged on its merits and no hard and fast rules can be laid down to embrace all cases. There is no necessity to-day to worry about blood grouping as the blood transfusion service for all the Services only use the blood from universal donors. Blood transfusion has made war surgery of the abdomen possible; without it the mortality resulting from resections would be about one hundred per cent. Not only does the Navy, Army and Air Force possess excellent blood transfusion services, but the same is true for the civilian population, and this is as it should be, for it may well be that the number of abdominal injuries amongst the civilians may be high, considering the method of warfare adopted by the Germans during the last six months. There is really not substitute to blood transfusion; intravenous gum and saline are worse than useless.

\section{Anæsthesia.}

The anæsthetic plays an important part in the treatment of abdominal injuries; without a good anæsthetic the surgeon cannot hope to perform a successful operation 
in these cases and the time factor will be greatly prolonged. The last war taught us that in these injuries speed in operating was all important, and this is impossible with a poor anæsthetic.

There can be no doubt that gas and oxygen anæsthesia is the anæsthetic of choice because so many of these cases are suffering from shock, but unless skilfully administered it is worse than useless, as it cramps the style of the surgeon and prolongs the time of the operation.

Open ether is an all round sound anæsthetic, especially if the patient is induced with a mixture of chloroform and ether. Induction with a C.E. mixture is much quieter than with open ether and the patients do not cough or struggle so much and it is quite safe, especially as most if not all the injured will have had an injection of morphia beforehand.

Spinal anæsthesia should never be used in these cases as the fall in blood pressure would in many cases be fatal, and the rolling the patient over or sitting him up in the acutely flexed position would increase the damage within the abdomen. Likewise the barbiturates are contraindicated in war injuries of the abdomen although they have already proved their worth in war wounds of the extremities where amputation is necessary.

\section{Operative Technique.}

It is necessary when dealing with war wounds of the abdomen to have adequate exposure, therefore the most useful incision is the paramedian one. Median incisions have been used and have their advocates, but they are more prone to hernia formation afterwards. Wounds in the abdominal wall should be carefully excised. Should the wounds be in the flank an iliac incision may be used in which the muscles in this region are divided; such an incision gives useful exposure. If by chance this is a wound in which both the thoracic and abdominal cavity are involved, then the surgeon must plan his incision so as to afford exposure to both these cavities.

Once the abdomen is opened arrest of hæmorrhage is of prime importance because it is the commonest cause of death in war wounds of the abdomen. Mesenteric vessels probably are the greatest danger and the root of the mesentery should always be carefully inspected. However, bleeding may come from the wall of the gut, the stomach, liver, spleen or omentum. Once the hæmorrhage is controlled blood transfusion can be started while the surgeon makes a routine examination of the small intestine.

\section{The small intestine.}

Commencing at the duodeno-jejunal junction the small bowel should be examined carefully but quickly loop by loop and returned to the abdominal cavity. Any perforation or wound of the small bowel requires suturing. Some holes in the intestine are very small and it seems difficult to believe that they were caused by a bullet at all, and these small perforations do not pout and therefore may be missed. Usually the mucous membrane is seen prolapsed through the wound in the bowel wall. (Fig. I.) Unless there are multiple perforations in a loop of bowel simple suture is sufficient, but excision is necessary where many perforations are found close together. A single layer of suture is quite sufficient, whereas a double layer may cause stenosis of the lumen. Resection of the bowel must be performed when there has been a separation of the bowel from the mesentery for a distance of more than half an inch. It must also be performed in cases of complete rupture of the bowel or where there has been intestinal infarction. 
Surgical commonsense is far more valuable in the treatment of wounds of the small intestine than any hard and fast rules which may from time to time be issued to the Services. In the last war there was no great difference in the frequency with which the jejunum and ileum were wounded, but there was no doubt that injuries

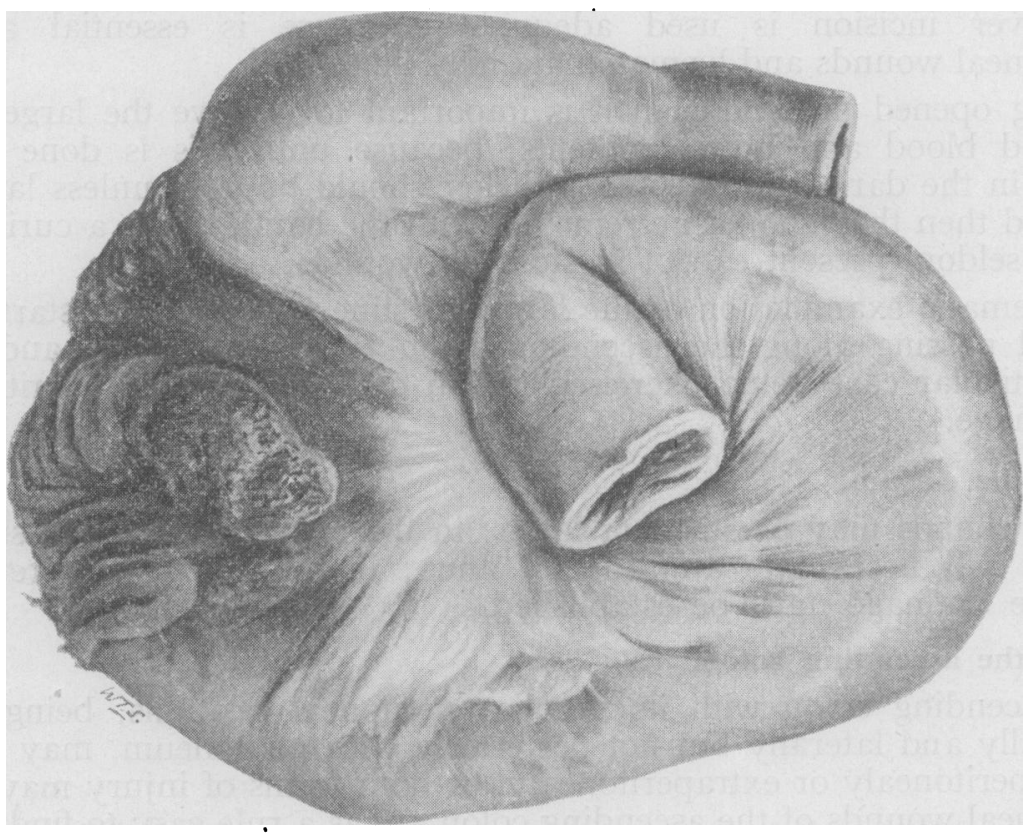

Fig. I.-(W.O. Collect. R.C.S. 887). G.S.W. of small intestine, and mesentery, from a patient who died of haemorrhoge from the mesentery and whose small intestine was extensively damaged and the adjacent mesentery injured.

of the ileum were more serious than those of the jejunum. This would be expected considering that the jejunum has a thicker wall and a much better blood supply and therefore lends itself to suture more easily.

The character of the wounds of the small intestine varies considerably, quite often a bullet may cause as severe an injury as a piece of high explosive shell.

\section{The large intestine.}

The large bowel injuries are in the main similar to those in the small intestine but there is always a greater tendency for escape of the contents of the large intestine. In many cases wounds of the large and small intestines co-exist. Colonic wounds are more grave not only because of the toxic contents of the large bowel, but because of the proximity of the retroperitoneal tissues and to the fact that the ascending and descending colons are not completely invested with peritoneum and therefore lack the protection of that membrane behind. Hæmatomata in the retrocolic and pericolic tissues are common and invariably become infected. Again infarction is more frequently met with in the large bowel wounds.

A paramedian incision is the best for large bowel injuries as a general exploration is necessary, but quite often the colon may be the only.part of the alimentary tract injured.

However, there is quite a lot to be said for the transverse incision in some cases ; it affords excellent approach to the ascending and descending colons, and the dangerous bare retroperitoneal aspect of the colon can easily be explored and, if 
necessary, an artificial anus can be established at the posterior end of an incision of this kind. Transverse incisions should cut across the rectus muscles at one of the tendinous intersections so that no damage is inflicted on the nerve supply of the abdominal muscles.

Whatever incision is used adequate exposure is essential as otherwise extraperitoneal wounds and hæmatomata may be missed.

Having opened the abdomen it is important to remove the large quantity of extravasated blood and bowel contents, because, until this is done the surgeon is working in the dark. If possible a sucker should be used unless large clots are present, and then they should be removed with the hand. It is a curious fact that free gas is seldom present except in stomach wounds.

A systematic examination of the large intestine is carried out starting with the cæcum and passing along the ascending colon to the transverse and descending colons, particular care being exercised not to overlook any retroperitoneal injury or hæmorrhage.

Wounds of the crecum.

Cæcal wounds may be small or large; in the former suture will suffice but, if large, they will necessitate an artificial anus; if associated with retroperitoneal hæmorrhage drainage must be established.

\section{Wounds of the ascending colon.}

The ascending colon with its peculiar peritoneal relations, being covered in front medially and laterally but not posteriorly with peritoneum, may be wounded either intraperitonealy or extraperitonealy, or both forms of injury may be present. Intraperitoneal wounds of the ascending colon are as a rule easy to find and suture ; however, the extraperitoneal wounds are often difficult to find as they may be small and valvular and are often obscured in the mass of retroperitoneal fat and hæmatoma. A colostomy may be necessary but should be avoided if possible.

\section{Wounds of the hepatic flexure.}

Wounds of the hepatic flexure are easy to deal with owing to the fact that this part of the colon is superficial in position; however, multiple wounds may be present in this region and these may necessitate a colostomy.

Wounds of the transverse colon.

The transverse colon may be injured in a variety of ways; it may be completely divided or a small perforation may be present. Quite often wounds of the transverse colon are associated with those of the stomach and small intestine and such multiple wounds are generally fatal and do not survive long enough for the surgeon to see them on the operating table. In wounds of the transverse colon alone excision is the method of choice but if this is impossible a colostomy should be performed as a life saving measure.

Wounds of the splenic flexure.

The splenic flexure is one of the most difficult regions of the large intestine to treat for gun shot injuries; it is high in position and deep in the abdomen and therefore it is difficult to expose and mobilise; hence colostomy is often necessary, and retroperitoneal hæmatomata are common and require drainage.

Wounds of the pelvic colon.

Wounds of the pelvic colon are easily dealt with if they occur in its upper portion, but lesions of the pelvirectal junction always demand colostomy. The 
surgeon is frequently confronted with the problem of suture versus colostomy in colon wounds and it is hard to dogmatise on this subject, but each case must be judged on its own merits and surgical common sense is of more value than brilliant technique.

Sir Cuthbert Wallace published some interesting figures after the Great War showing colon injuries uncomplicated with wounds of other hollow viscera. Some of the injuries were treated by simple suture, the others by colostomy.

\begin{tabular}{|lll|c|c|c|}
\hline \multicolumn{2}{|c|}{ Site of Lesion } & Sutured & Colostomy & Total \\
\cline { 1 - 3 } Cæcum .. & $\ldots$ & 13 & 3 & 16 \\
Ascending colon & $\ldots$ & 25 & 11 & 36 \\
Hepatic flexure & $\ldots$ & 16 & 8 & 24 \\
Transverse colon &.. & 13 & 2 & 15 \\
Splenic flexure &.. & 10 & 8 & 18 \\
Descending colon .. & 9 & 8 & 17 \\
Pelvic colon &.. & 16 & 7 & 23 \\
\hline
\end{tabular}

Wounds of the rectum.

Wounds of the rectum are very fatal, especially if complicated by wounds of the buttocks, perineum and ischio-rectal fossæ. Such wounds are also liable to be further complicated with fractures of the pelvic girdle. Gas gangrene is especially liable to develop in wounds of the gluteal region. Wide excision of the wound and a colostomy are often required as a life saving procedure. Transverse colostomy is the method of choice as it is easier to manage; a better belt can be fitted and finally it is easier to close at a later date should this be considered safe.

\section{Drainage of the abdomen.}

The question of drainage in abdominal wounds is an all important one and yet one which may be a fruitful source of disagreement. The last war taught the surgeon that soiling of the peritoneal cavity by bowel contents did not always require drainage and many cases did well without it. However, all cases of retroperitoneal infection demanded drainage.

Closure of the abdomen after operations for gun-shot wounds may have to be hurried owing to the fact that the utmost speed may be necessary, and so sutures passing through the whole thickness of the abdominal wall may have to be used. However, whenever possible a separate peritoneal closure should be aimed at and then through and through sutures for the muscles and separate sutures for the skin.

\section{CONCLUSION.}

The present war will probably provide a large number of abdominal casualties, and those due to high explosives will probably be more frequent than those due to bullet wounds. However, we have, both at home and abroad, ample facilities for dealing with hæmorrhage with our blood transfusion services, which should prove of real value. 